\title{
A Mathematical Model for Interfacial Defects in Snow Layers
}

\author{
Aditya Khanna and Andrei Kotousov
}

\begin{abstract}
Existing theories of snow slab failure postulate that failure initiates with the propagation of an interfacial defect located between snow layers with similar mechanical properties. In this paper, we develop a simplified model for failure initiation when there is a strong mismatch in material properties of the bounding snow layers. The crack problem is formulated in terms of the distributed dislocation technique and the governing integral equations are solved using the Gauss-Chebyshev quadrature method. We obtain theoretical dependence of the mode II stress intensity factor upon the mismatch in material properties, slope angle and snow layer thickness.
\end{abstract}

Index Terms-Crack closure, distributed dislocation technique, interfacial crack, snow slab avalanche.

\section{INTRODUCTION}

Snow avalanches down mountain slopes either gradually (loose snow avalanche) or catastrophically (snow slab avalanche). Loose snow avalanches are analogous to the rotational slip of cohesion-less sand and typically occur at mountain slopes exceeding $45^{\circ}$, preventing the accumulation of excessive snow at these relatively steep slopes. Slab avalanches on the other hand, occur at moderately steep slopes in the range of $30^{\circ}-45^{\circ}$ and release a large volume of cohesive snow (the slab) at once [1], [2]. Dry snow slab avalanches are a major hazard, endangering residents and infrastructure when triggered naturally or recreationists in the case of human triggered events.

It is well established that a dry snow slab avalanche is the result of shear failure along a weak layer or interface underlying the snowpack or slab followed by the tensile failure at the top of the slab (crown) and compressive failure at the bottom of the slab (staunchwall) [1]-[3] (Fig. 1). Several mechanical models have been presented in the past to predict avalanche release conditions [4]-[9]. These models invariably consider the situation where the slip surface is situated between two layers of snow with similar material properties.

In this paper, we consider the scenario when there is significant mismatch in material properties of the bounding layers. Such a situation may arise when the preexisting defect lies between the snow layer and the rocky substratum, or between two snow layers with different microstructures. The defect is modeled as an interfacial crack between a layer and a half plane. The layer represents the overlying snow slab and the half plane represents the underlying slope. The crack is

Manuscript received May 6, 2015; revised July 23, 2015.

The authors are with the School of Mechanical Engineering, the University of Adelaide, Adelaide, SA 5005, Australia (e-mail: aditya.khanna@adelaide.edu.au, andrei.kotousov@adelaide.edu.au). modeled using the distributed dislocation technique [10], [11], and the effect of slab thickness and crack closure are incorporated are incorporated into the governing singular integral equations using the method described in [12]-[16]. The governing equations are solved numerically using the Gauss-Chebyshev quadrature [17]-[20] and the dependence of the energy release rate on material mismatch, slope angle and snow layer thickness is obtained.

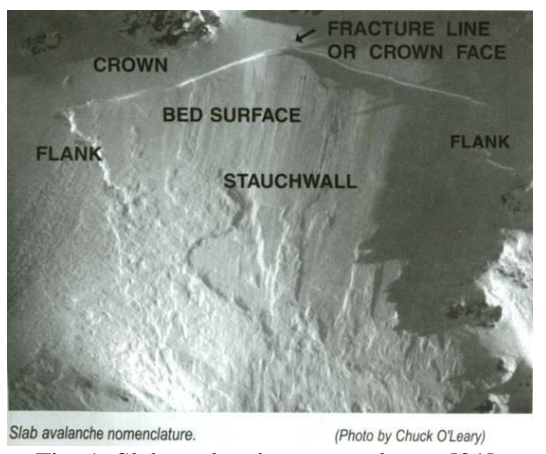

Fig. 1. Slab avalanche nomenclature [21].

\section{PROBLEM FORMULATION}

The crack problem posed is illustrated in Fig. 2. The layer, of thickness $t$, is perfectly bonded to the substrate everywhere except for the location of the crack of length $2 L$. The $x$-axis is taken to be the layer-substrate interface. In the absence of the interfacial crack, the snow loading produces uniform normal and shear tractions along the location of the interfacial crack, given by

$$
\begin{gathered}
T(x)=T=-\rho g t \cos ^{2} \phi, \\
Q(x)=Q=-\rho g t \sin \phi \cos \phi,
\end{gathered}
$$

respectively. In Eqs. (1)-(2), $\rho$ is the density of the snow slab, $g$ is the acceleration due to gravity, $t$ is the thickness of the snow slab and $\phi$ is the slope angle.

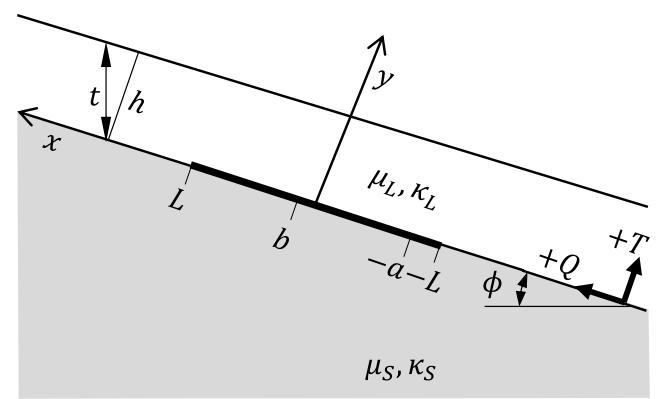

Fig. 2. 2D slab release model showing an interfacial crack between the slab layer and the substratum. 
The crack will be partially closed and at least two contact zones develop in the vicinity of the crack tips, as discussed previously. The two contact zones occupy the interval $(-L,-a)$ and $(b, L)$, while $(-a, b)$ is the interval over which an open zone or gap exists between the layer and substrate. The opening and tangential shift along the crack can be defined as

$$
\begin{aligned}
& g(x)=u_{y}\left(x, 0^{+}\right)-u_{y}\left(x, 0^{-}\right), \\
& h(x)=u_{x}\left(x, 0^{+}\right)-u_{x}\left(x, 0^{-}\right),
\end{aligned}
$$

respectively. The gap can be modeled by a distribution of climb dislocations with density $B_{y}(x)$, and the tangential shift by a distribution of glide dislocations with density $B_{x}(x)$ so that

$$
B_{y}(x)=-\frac{d g}{d x}, \quad B_{x}(x)=-\frac{d h}{d x}
$$

Note that $B_{y}(x)$ is defined over the interval $(-a, b)$ and $B_{x}(x)$ is defined over the interval $(-L, L)$ and they are zero everywhere else. The governing integral equations for the interfacial crack can be written as [11]-[14].

$$
\begin{gathered}
-\beta B_{x}(x)+\frac{1}{\pi} \int_{-a}^{+b} \frac{B_{y}(\xi)}{x-\xi} d \xi+\frac{1}{\pi} \int_{-a}^{+b} B_{y}(\xi) K_{y y y}(x, \xi) d \xi \\
+\frac{1}{\pi} \int_{-L}^{+L} B_{x}(\xi) K_{x y y}(x, \xi) d \xi=-\frac{T}{C}, \quad-a<x<b, \\
-\beta B_{y}(x)+\frac{1}{\pi} \int_{-L}^{+L} \frac{B_{x}(\xi)}{x-\xi} d \xi+\frac{1}{\pi} \int_{-L}^{+L} B_{x}(\xi) K_{x x y}(x, \xi) d \xi \\
+\frac{1}{\pi} \int_{-a}^{+b} B_{y}(\xi) K_{y x y}(x, \xi) d \xi=-\frac{Q}{C}, \quad-L<x<L,
\end{gathered}
$$

where $C$ is the effective bimaterial modulus defined as

$$
C=\frac{2 \mu_{L}}{\left(\kappa_{L}+1\right)} \frac{1-\alpha}{1-\beta^{2}}=\frac{2 \mu_{S}}{\left(\kappa_{S}+1\right)} \frac{1+\alpha}{1-\beta^{2}},
$$

In terms of Dundur's parameters $\alpha$ and $\beta$,

$$
\begin{array}{r}
\alpha=\frac{\mu_{L}\left(\kappa_{S}+1\right)-\mu_{S}\left(\kappa_{L}+1\right)}{\mu_{L}\left(\kappa_{S}+1\right)+\mu_{S}\left(\kappa_{L}+1\right)}, \\
\beta=\frac{\mu_{L}\left(\kappa_{S}-1\right)-\mu_{S}\left(\kappa_{L}-1\right)}{\mu_{L}\left(\kappa_{S}+1\right)+\mu_{S}\left(\kappa_{L}+1\right)} .
\end{array}
$$

The elastic constants $\mu_{L}, \kappa_{L}$ correspond to the layer and $\mu_{S}, \kappa_{S}$ correspond to the substratum. The kernels $K_{i j k}(x, \xi)$ are regular bounded functions describing the influence of the layered structure, and can be obtained from [12]. In addition, the following uniqueness conditions must be imposed

$$
\int_{-a}^{b} B_{y}(\xi) d \xi
$$

$$
\int_{-L}^{L} B_{x}(\xi) d \xi
$$

The singular integral Eqs. (6) and (7), along with Eqs. (10) -(11) are sufficient to obtain the solution for $B_{x}(x)$ and $B_{y}(x)$ as well as the unknown constants $a$ and $b$.

\section{NUMERICAL RESULTS}

Here, we investigate the effect of slope angle and the snow layer thickness on various physical quantities of interest. We take shear modulus of snow, $\mu_{L}=0.42 \mathrm{MPa}$, Kolosov's constant for snow, $\kappa_{L}=2.2$ and Dundur's parameters $\alpha=-1$ and $\beta=-0.5$, which corresponds to the case of a rigid substratum. The method of obtaining the solution for the coupled system of governing integral equations is described in [11], [14]-[20] and is omitted here. Recalling that one contact zone is expected to be infinitesimal, $\mathrm{O}\left(10^{-7} L\right)$ and the other large in extent, $\mathrm{O}(L)$, we fix $\beta Q>0$. This has the effect that the large contact zone always appears at the right tip [11], [14] and the discretization procedure is simplified without loss of generality. To further simplify the numerical procedure, we interchange the roles of $b / L$ and $T / Q$. Thus, we treat $b / L$ as known (specified) and derive the value of $T / Q$ as a part of the solution.
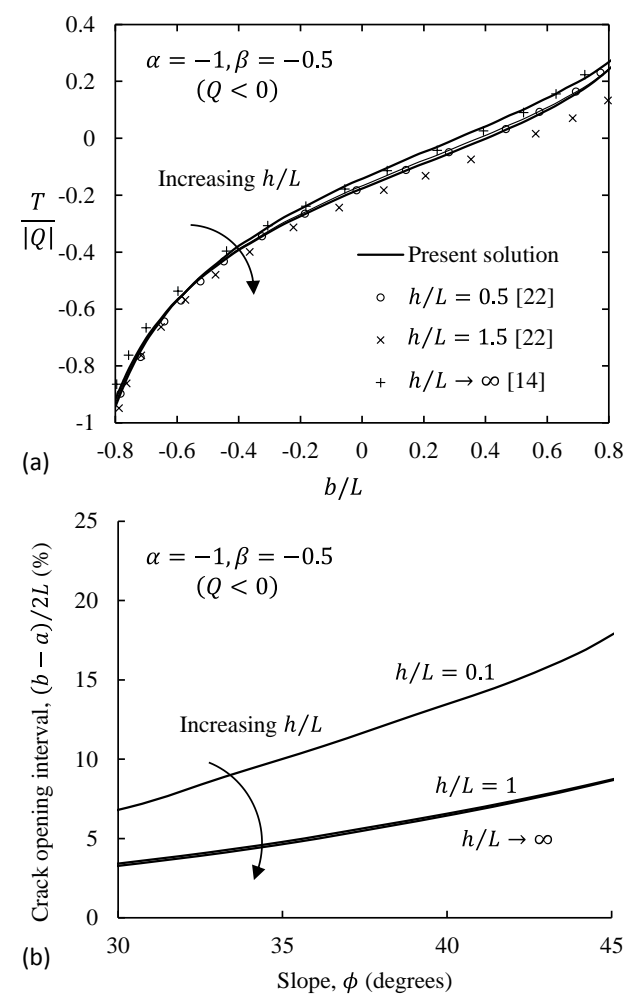

Fig. 3. (a) Validation of the present solution (b) Dependence of the crack opening interval on the slope and normalized layer thickness.

In Fig. 3(a), we compare the obtained dependence of $T / Q$ upon $b / L$ to previously obtained results. Both sets of results demonstrate that the layer thickness, $h / L$ has little effect on the obtained dependence over the interval $h / L \in[0.5, \infty)$. 
The dependence of $T / Q$ on $h / L$ obtained in [22] for $h / L \rightarrow \infty$ is out of line with the present results and results provided in [14]. This discrepancy could be attributed to the numerical solution procedure utilized in [22]. Overall, the present numerical results are in fair agreement with previously obtained results, serving as a validation of the present approach.

Fig. 3(b) shows the dependence of the crack opening interval upon the slope angle. The slope angle can be obtained from Eqs. (1)-(2) as $\phi=\cot ^{-1}(T / Q)$ and the length of the opened region of the crack can be obtained as $(b-a)$ from Fig. 2. It is found that under shear and compressive loading which would prevail at typical slope angles $\left(30^{0}-45^{0}\right)$, the crack would primarily remain closed, with a very small opened zone. The length of the opened zone is expected to increase with increasing slope and increasing crack length (for a given layer thickness). The large contact zone also implies that the effect of friction must be incorporated in the accurate analysis of the problem under consideration.
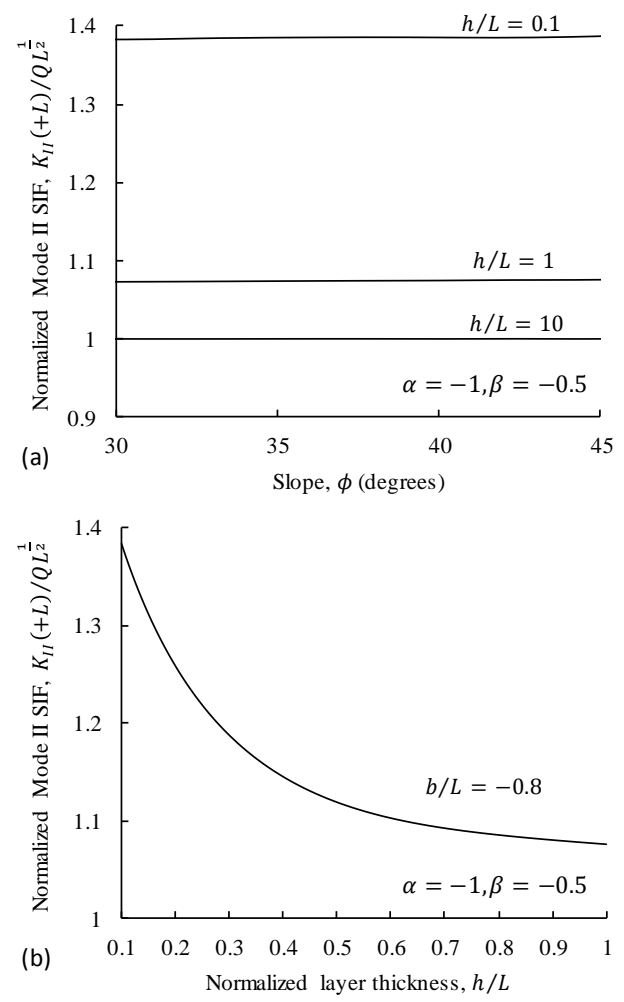

Fig. 3. Dependence of the normalized Mode II SIF on (a) slope angle and (b) normalized layer thickness.

The mode II stress intensity factor (SIF) for an interfacial crack with contact zones is defined by [22], [23] as

$$
K_{I I}( \pm L)= \pm C L^{1 / 2} \lim _{s \rightarrow \pm 1}\left\{\left(1-s^{2}\right)^{1 / 2} B_{x}(s)\right\}
$$

and the strain energy release rate can be related to the mode II SIF according to [23]

$$
G=\frac{\pi K_{I I}^{2}}{4 C} .
$$

Fig. 4(a) shows the dependence of the normalized Mode II stress intensity factor at the right crack tip, $(x=+L)$ upon the slope angle for various values of the normalized layer thickness, $h / L$. It is observed for all values of the ratio $h / L$, the parameter $K_{I I} / Q L^{1 / 2}$ is rather insensitive to variation in the slope angle $\phi$, within the range considered. At these angles, the crack is mostly closed (Fig. 3(b) ).

Taking advantage of the weak dependence of the normalized Mode II SIF on the slope angle, the value of $b / L$ was fixed at 0.8 for further calculations. Decreasing layer thickness implies that the interfacial crack experiences increased stress concentration due to the presence of a free surface at $y=h$. Hence, the normalized Mode II SIF increases in magnitude with decreasing normalized layer thickness, as shown in Fig. 4(b).

\section{CONCLUSION}

A mathematical model is presented for an interfacial crack present between a snow layer and a rigid substratum. It is shown that under the combined shear and compressive loading, the interfacial crack remains mostly closed which suggests that effects of friction need to be incorporated in more sophisticated future models. The dependence of the mode II stress intensity factor upon the slope angle and layer thickness is also presented. Although the problem has not been exhausted and a complete parametric study was not undertaken, it is hoped that its salient features have been sufficiently presented.

\section{REFERENCES}

[1] J. Schweizer, J. B. Jamieson, and M. Schneebeli, "Snow avalanche formation," Reviews of Geophysics, vol. 41, no. 4, pp. 1-25, December 2003.

[2] R. I. Perla and E. R. LaChapelle, "A theory of snow slab failure," Journal of Geophysical Research, vol. 75, no. 36, pp. 7619-7627, December 1970.

[3] E. H. Bair, R. Simenhois, K. Birkeland, and J. Dozier, "A field study on failure of storm snow slab avalanches," Cold Regions Science and Technology, vol. 79-80, pp. 20-28, August 2012.

[4] D. M. McClung, "Shear fracture precipitated by strain softening as a mechanism of dry slab avalanche release," Journal of Geophysical Research: Solid Earth, vol. 84, no. B7, pp. 3519-3526, July 1979.

[5] D. M. McClung, "Fracture mechanical models of dry slab avalanche release," Journal of Geophysical Research: Solid Earth, vol. 86, no. B11, pp. 10783-10790, November 1981.

[6] J. Schweizer, "The influence of the layered character of snow cover on the triggering of slab avalanches," Annals of Glaciology, vol. 18, pp. 193-198, 1993.

[7] B. M. Chiaia, P. Cornetti, and B. Frigo, "Triggering of dry snow slab avalanches: stress versus fracture mechanical approach," Cold Regions Science and Technology, vol. 53, no. 2, pp. 170-178, July 2008.

[8] F. Louchet, "A simple model for dry snow slab avalanche triggering," Comptes Rendus de l'Académie des Sciences - Series IIA — Earth and Planetary Science, vol. 330, no. 12, pp. 821-827, June 2000.

[9] J. Heierli, K. W. Birkeland, R. Simenhois, and P. Gumbsch, "Anticrack model for skier triggering of slab avalanches," Cold Regions Science and Technology, vol. 65, no. 3, pp. 372-381, March 2011.

[10] B. Bilby and J. Eshelby, "Dislocations and the theory of fracture," in Fracture: An Advanced Treatise, H. Liebowitz, Ed., vol. 1, New York: Academic Press, 1968, pp. 99-182.

[11] D. A. Hills, P. A. Kelly, D. N. Dai, and A. M. Korsunsky, Solution of Crack Problems: The Distributed Dislocation Technique, Dordrecht, The Netherlands: Kluwer Academic Publishers, 1996, ch. 4, pp. 107-126. 
[12] F. Erdogan and G. Gupta, "The stress analysis of multi-layered composites with a flaw," International Journal of Solids and Structures, vol. 7, no. 1, pp. 39-61, January 1971.

[13] F. Erdogan and G. D. Gupta, "Layered composites with an interface flaw," International Journal of Solids and Structures, vol. 7, no. 8, pp. 1089-1107, August 1971.

[14] M. Comninou and J. Dundurs, "Partial closure of cracks at the interface between a layer and a half space," Engineering Fracture Mechanics, vol. 18, no. 2, pp. 315-323, 1983.

[15] A. Khanna and A. Kotousov, "Stress analysis of a crack in a fiber-reinforced layered composite," Composite Structures, vol. 118 , pp. 139-148, December 2014.

[16] A. Khanna and A. Kotousov, "Controlling the height of multiple hydraulic fractures in layered media," SPE Journal, SPE-176017-PA May 2015.

[17] A. Khanna, L. B. Neto and A. Kotousov, "Effect of residual opening on the inflow performance of a hydraulic fracture," International Journal of Engineering Science, vol. 74, pp. 80-90, January 2014.

[18] A. Kotousov, L. B. Neto and A. Khanna, "On a rigid inclusion pressed between two elastic half spaces," Mechanics of Materials, vol. 68, pp. 38-44, January 2014.

[19] L. B. Neto, A. Khanna, and A. Kotousov, "Conductivity and performance of hydraulic fractures partially filled with compressible proppant packs," International Journal of Rock Mechanics and Mining Sciences, vol. 74, pp. 1-9, February 2015.

[20] L. B. Neto and A. Khanna, "The performance of hydraulic fractures partially filled with compressible proppant," Australian Journal of Multi-disciplinary Engineering, vol. 10, no. 2, pp. 185-197, 2013.

[21] J. A. Fredston and D. Fesler, Snow Sense: A Guide to Evaluating Snow Avalanche Hazard, Alaska Mountain Safety Center, Inc., 1999.

[22] M. Comninou and D. Schmueser, "The interface crack in a combined tension-compression and shear field," Journal of Applied Mechanics, vol. 46, pp. 345-348, June 1979.

[23] M. Comninou, "The interface crack," Journal of Applied Mechanics, vol. 44, pp. 631-636, December 1977.

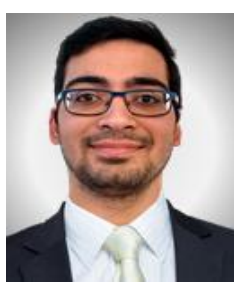

Aditya Khanna is currently a $\mathrm{PhD}$ candidate at the University of Adelaide, Adelaide, Australia. Khanna obtained his BS degree in mechanical engineering in 2012 from the same institution. His major field of study is theoretical fracture mechanics and modelling of natural fracture phenomena.

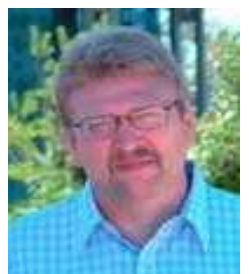

in these areas
Andrei Kotousov earned a $\mathrm{PhD}$ degree at the Russian Academy of Sciences, and is currently an associate professor at the School of Mechanical Engineering at the University of Adelaide. He has broad experience in theoretical and experimental fracture mechanics, biomechanics, structural-health monitoring, and composite materials. Kotousov has produced more than 150 peer-reviewed publications 